\title{
Synthesis, Structures, Solution Behavior, and Reactions of Thiolato-Bridged Diruthenium Carbonyl Phosphine Complexes
}

\author{
Kom-Bei Shiu* \\ Department of Chemistry, National Cheng Kung University, Tainan, Taiwan 701 \\ Sue-Lein Wang and Fen-Ling Liao \\ Department of Chemistry, National Tsing Hua University, Hsinchu, Taiwan 300 \\ Michael Y. Chiang \\ Department of Chemistry, National Sun Yat-Sen University, Kaohsiung, Taiwan 804 \\ Shie-Ming Peng and Gene-Hsiang Lee \\ Department of Chemistry, National Taiwan University, Taipei, Taiwan 106 \\ J u-Chun Wang and Lin-Shu Liou \\ Department of Chemistry, Soochow University, Taipe, Taiwan 111
}

Recei ved November 13, 1997

\begin{abstract}
Dinuclear thiolato-bridged complexes $\left[\mathrm{Ru}_{2}(\mathrm{CO})_{4}\left(\mu-\mathrm{SR}_{2}\left(\mathrm{PR}_{3}^{\prime}\right)_{2}\right]\left(\mathrm{R}^{\prime}=\mathrm{Ph}, \mathrm{Me}\right)\right.$ have been readily prepared from the reaction of $\left[\mathrm{Ru}_{2}(\mathrm{CO})_{4}(\mathrm{MeCN})_{4}\left(\mathrm{PR}_{3}^{\prime}\right)_{2}\right]\left[\mathrm{BF}_{4}\right]_{2}\left(\mathrm{R}^{\prime}=\mathrm{Ph}(\mathbf{1})\right.$, Me (2)) with $\mathrm{RSH}\left(\mathrm{R}={ }^{\mathrm{t}} \mathrm{Bu},{ }^{\mathrm{i}} \mathrm{Pr}, \mathrm{Ph}\right)$ and $\mathrm{Et}_{3} \mathrm{~N}$ at ambient temperature. Although only the syn form of $\left[\mathrm{Ru}_{2}(\mathrm{CO})_{4}(\mu-\mathrm{S} \mathrm{Bu})_{2}\left(\mathrm{PPh}_{3}\right)_{2}\right](3)$ and only the anti form of $\left[\mathrm{Ru}_{2}(\mathrm{CO})_{4}(\mu-\mathrm{SPh})_{2}\left(\mathrm{PMe}_{3}\right)_{2}\right](6)$ were found, an equilibrium mixture of both the syn (isomer $\mathbf{A}$ ) and anti (isomer $\mathbf{B}$ ) forms was present in solution for $\left[\mathrm{Ru}_{2}(\mathrm{CO})_{4}(\mu-\mathrm{Si} \mathrm{Pr})_{2}\left(\mathrm{PPh}_{3}\right)_{2}\right](\mathbf{4})$ and $\left[\mathrm{Ru}_{2}(\mathrm{CO})_{4}(u-\mathrm{SPh})_{2}\left(\mathrm{PPh}_{3}\right)_{2}\right](\mathbf{5})$. The spectral data support that the syn form (4A) is the major isomer of $\mathbf{4}$, while the anti form (5B) is the major isomer of $\mathbf{5}$. The two thiolato bridges are located cis to the two phosphine ligands in solid-state structures 3, 4A, and 5B, but they are located cis to only one of the two ligands whereas they are trans to the other in structure 6 . I odination of 3-6 gave one identical product $\left[\mathrm{Ru}_{2}(\mathrm{CO})_{4}(\mu-\mathrm{SR})_{2} \mathrm{I}_{2}\left(\mathrm{PR}_{3}^{\prime}\right)_{2}\right]\left(\mathrm{R}^{\prime}=\mathrm{Ph}, \mathrm{R}={ }^{\mathrm{t}} \mathrm{Bu}(\mathbf{7}),{ }^{\mathrm{i}} \mathrm{Pr}(\mathbf{8}), \mathrm{Ph}(\mathbf{9})\right.$; $\mathrm{R}^{\prime}=\mathrm{Me}, \mathrm{R}=\mathrm{Ph}(\mathbf{1 0})$ ). Both spectral and structural evidence shows that $\mathbf{7}-\mathbf{1 0}$ exist in the syn form with no Ru-Ru bonding interaction. The relative orientation of the two thiolato bridges with respect to the two phosphine ligands present in 6 remains in structure 10, in spite of the change from anti to syn. The reactions of the diiodide complexes $\left[\operatorname{Ru}_{2}(C O)_{4}(\mu-\right.$ $\left.\mathrm{SR})_{2} \mathrm{I}_{2}\left(\mathrm{PR}_{3}^{\prime}\right)_{2}\right]$ with $\mathrm{R}^{\prime \prime} \mathrm{S}$ anions give either $\left[\mathrm{Ru}_{2}(\mathrm{CO})_{4}(\mu-\mathrm{SR})_{2}\left(\mathrm{PR}_{3}^{\prime}\right)_{2}\right]$ and $\mathrm{R}^{\prime \prime} \mathrm{SSR}^{\prime \prime}$ or $\left[\mathrm{Ru}_{2^{-}}\right.$ $\left.(\mathrm{CO})_{4}(\mu-\mathrm{SR})_{2}\left(\mathrm{SR}^{\prime \prime}\right)_{2}\left(\mathrm{PR}_{3}^{\prime}\right)_{2}\right]$.
\end{abstract}

\section{Introduction}

Transition-metal complexes with sulfur ligands are of significant interest not only because they often display unusual structures and novel reactivity ${ }^{1}$ but also because they can serve as synthetic anal ogues for the active sites of metalloprotein, ${ }^{2}$ and show some relevance to metal sulfide hydrodesulfurization and demercuration catalysts. ${ }^{3}$

* To whom correspondence should be addressed. Fax: (+886) 6274 0552. E-mail: kbshiu@mail.ncku.edu.tw.

(1) For recent reviews, see: (a) Holm, R. H.; Ciurli, S.; Weigel, J . A. Prog. I norg. Chem. 1990, 38, 1. (b) Krebs, B.; Henkel, G. Angew. Chem., Int. Ed. Engl. 1991, 30, 769. (c) Shibahara, T. Coord. Chem. Rev. 1993, 123, 73. (d) Saito, T. In Early Transition Metal Clusters with $\pi$-Donor Ligands; Chisholm, M. H., Ed.; VCH: New York, 1995; Chapter 3. (e) Dance, I.; Fisher, K. Prog. Inorg. Chem. 1994, 41, 637. (f) Stiefel, E. I. Ed. Transition Metal Sulfur Chemistry; American Chemical Society: Washington, DC, 1996.
Diiron thiolato-bridged complexes, $\left[\mathrm{Fe}_{2}(\mathrm{CO})_{4}(u-\mathrm{SR})_{2} \mathrm{~L}_{2}\right]$ $(\mathrm{L}=\mathrm{CO}$, phosphine, phosphite, one or one-half diphosphine or -arsineligand; R = alkyl, phenyl, or aryl), have been studied extensively in terms of synthesis, reactivity, and spectroscopic measurements. ${ }^{4}$ The existence of two isomers, syn and anti, with a common ${ }^{4-6}$ arrangement for the two axially coordinated ligands $\mathrm{L}=$ phosphine, trans to the metal-metal bond (Chart 1),

(2) (a) Coucouvanis, D. Adv. Inorg. Chem. 1992, 38. 1. (b) Coucouvanis, D. In Molybenum Enzymes, Cofactors, and Model systems; Stiefel, E. I., Coucouvanis, D., Newton, W. E., Eds.; American Chemical Society: Washington, DC, 1993; p 304. (c) Rees, D. C.; Chan, M. K.; Kim, J. Adv. Inorg. Chem. 1994, 40, 89.

(3) (a) Angelici, R. J. Acc. Chem. Res. 1988, 21, 387. (b) Chen, J .; Daniels, L. M.; Angelici, R. J . J . Am. Chem. Soc. 1990, 112, 199. (c) Wiegand, B. C.; Friend, C. M. Chem. Rev. 1992, 92, 491. (d) Riaz, V.; Curnow, O. J .; Curtis, M. D. J. Am. Chem. Soc. 1994, 116, 4357. (e) Bianchini, C.; Meli, A. J . Chem. Soc., Dalton Trans. 1996, 801. (f) Hill, A. F.; Wilton-Ely, J . D. E. T. Organometallics 1997, 16, 4517. 


\section{Chart 1}

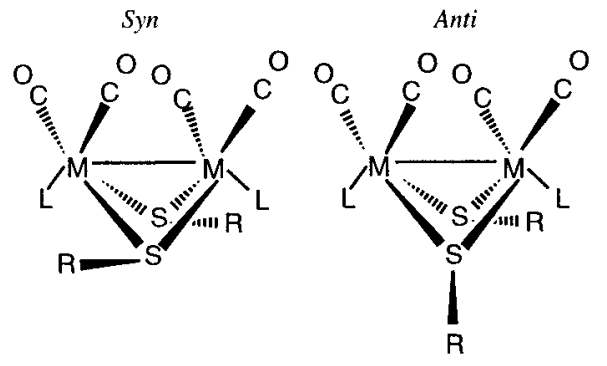

and equilibrated isomerization favoring one isomer or the other were recognized for some types of compounds. Thermal oxidative and reductive decompositions yielding sulfides, disulfides, and thiols were also reported. In contrast the chemistry of diruthenium thiolatobridged complexes are still relatively unexplored. ${ }^{5}$

In this paper, we present the following new information: (1) the reactions between $\left[\mathrm{Ru}_{2}(\mathrm{CO})_{4}(\mathrm{MeCN})_{4^{-}}\right.$ $\left.\left(\mathrm{PR}_{3}^{\prime}\right)_{2}\right]\left[\mathrm{BF}_{4}\right]_{2}\left(\mathrm{R}^{\prime}=\mathrm{Ph}(\mathbf{1}), \mathrm{Me}(\mathbf{2})\right)$ and two thiolate anions via $\mathrm{RSH} / \mathrm{Et}_{3} \mathrm{~N}$ can occur even at ambient temperature to afford the $\mathrm{Ru}-\mathrm{Ru}$ singly bonded compounds $\left[\mathrm{Ru}_{2}(\mathrm{CO})_{4}(\mu-\mathrm{SR})_{2}\left(\mathrm{PR}_{3}{ }_{3}\right)_{2}\right](3-6)\left(\mathrm{R}={ }^{\mathrm{t}} \mathrm{Bu}, \mathrm{i} \mathrm{Pr}, \mathrm{Ph} ; \mathrm{R}^{\prime}=\right.$ $\mathrm{Ph}, \mathrm{Me}) ;(2)$ the facile interconversion between syn and anti forms of $\left[\mathrm{Ru}_{2}(\mathrm{CO})_{4}(\mu-\mathrm{SiPr})_{2}\left(\mathrm{PPh}_{3}\right)_{2}\right](\mathbf{4})$ and $\left[\mathrm{Ru}_{2}-\right.$ $\left.(\mathrm{CO})_{4}(u-\mathrm{SPh})_{2}\left(\mathrm{PPh}_{3}\right)_{2}\right]$ (5) is observed at ambient temperature in solution; (3) the first crystallographically characterized syn and anti forms of the diruthenium thi olato-bridged carbonyl phosphine complexes are presented, adopting either a common geometry in 3-5, with the two thiolato bridges cis to the two phosphine groups observed previously in diiron thiolato-bridged complexes, ${ }^{4 i}$ or an unprecedented geometry, with the two thiolato bridges cis to one but trans to the other phosphine ligand in 6; (4) iodination of 3-6 affords the single diiodide adducts (7-10) all in the syn form, where the unique geometry observed in $\mathbf{6}$ is retained in its diiodide adduct 10; and (5) the reactions of $\left[\mathrm{Ru}_{2}(\mathrm{CO})_{4^{-}}\right.$ $\left.(\mu-\mathrm{SR})_{2} \mathrm{I}_{2}\left(\mathrm{PR}_{3}^{\prime}\right)_{2}\right]$ with $\mathrm{R}^{\prime \prime} \mathrm{S}^{-}$via $\mathrm{R}^{\prime \prime} \mathrm{SH} / \mathrm{Et}_{3} \mathrm{~N}$ produce either the substituted product $\left[\mathrm{Ru}_{2}(\mathrm{CO})_{4}(\mu-\mathrm{SR})_{2}\left(\mathrm{SR}^{\prime \prime}\right)_{2^{-}}\right.$ $\left.\left(\mathrm{PR}_{3}{ }_{3}\right)_{2}\right]$ or the reductive-deiodination products [Ru $2_{2-}^{-}$ $\left.(\mathrm{CO})_{4}(u-\mathrm{SR})_{2}\left(\mathrm{PR}_{3}{ }_{3}\right)_{2}\right]$ with apparently external $\mathrm{R}^{\prime \prime} \mathrm{S}^{-}$ being oxidized into R"SSR".

(4) (a) Dahl, L. F.; Wei, C.-H. Inorg. Chem. 1963, 2, 328. (b) Hieber W.; Kaiser, K. Chem. Ber. 1969, 102, 4043. (c) Crow, J . P.; Cullen, W. R. Can. J . Chem. 1971, 49, 2948. (d) De Beer, J. A.; Haines, R. J .J. Chem. Soc. A 1971, 3271. (e) Maresca, L.; Greggio, F.; Sbrignadello, G.; Bor, G. Inorg. Chim. Acta 1971, 5, 667. (f) De Beer, J . A., Haines, R. J . J . Organomet. Chem. 1972, 36, 297. (g) De Beer, J . A.; Haines, R. J . J . Organomet. Chem. 1972, 37, 173. (h) Ellgen, P. C.; Gerlach, J N. Inorg. Chem. 1973, 12, 2526. (i) Borgne, G. L.; Grandjean, D. Mathieu, R.; Poilblanc, R. J . Organomet. Chem. 1977, 131, 429. (j) Nametkin, N. S.; Tyurin, V. D.; Kukina, M. A. J . Organomet. Chem 1978, 149, 355. (k) Shriver, D. F.; Whitmire, K. H. In Comprehensive Organometallic Chemistry; Wilkinson, G., Stone, F. G. A., Abel, E. W. Eds.; Pergamon: Oxford, England, 1982; Vol. 4, Chapter 31, p 280. (I) Hawker, P. H.; Twigg, M. V. In Comprehensive Coordination Chemistry; Wilkinson, G. W., Gillard, R. D., Eds.; Pergamon: Oxford, England, 1987; Vol. 4. Chapter 44.1, p 1238. (m) Whitmire, K. H, In Comprehensive Organometallic Chemistry II; Abel, E. W., Stone, F. G. A., Wilkinson, G., Eds.; Pergamon: Oxford, England, 1995; Vol. 7, Chapter $1, \mathrm{p} 65$.

(5) (a) Andreu, P. L.; Cabeza, J . A.; Riera, V.; Robert, F.; J eannin, Y. J . Organomet. Chem. 1989, 372, C15. (b) Shiu, K.-B.; Li, C.-H.; Chan T.-J .; Peng, S.-M.; Cheng, M.-C.; Wang, S.-L.; Liao, F.-L. Organome tallics 1995, 14, 524. (c) Soler, J .; Ros, J .; Carrasco, M. R.; Ruiz, A.; Alvarez-Larena, A.; Piniella, J. F. Inorg. Chem. 1995, 34, 6211.

(6) Andreu, P. L.; Cabeza, J. A.; Miguel, D.; Riera, V.; Villa, M. A . Garcia-Granda, S. J . Chem. Soc., Dalton Trans. 1991, 533.

\section{Experimental Section}

All solvents were dried and purified by standard methods (ethers, paraffins, and arenes from potassium with benzophenone as indicator; halocarbons and acetonitrile from $\mathrm{CaH}_{2}$ and alcohols from the corresponding alkoxide) and were freshly distilled under nitrogen immediately before use. All reactions and manipulations were carried out in standard Schlenk ware, connected to a switchable double manifold providing vacuum and nitrogen. Reagents were used as supplied by Aldrich. ${ }^{1 \mathrm{H}}$ and ${ }^{31} \mathrm{P}$ NMR spectra were measured on a Brueker AMC-400 $\left({ }^{1} \mathrm{H}, 400 \mathrm{MHz} ;{ }^{31} \mathrm{P}, 162 \mathrm{MHz} ;{ }^{13} \mathrm{C}, 100 \mathrm{MHz}\right) \mathrm{NMR}$ spectrometer. ${ }^{1} \mathrm{H}$ chemical shifts ( $\delta$ in ppm, $J$ in hertz) are defined as positive downfield relative to internal $\mathrm{MeSi}_{4}$ (TMS) or the deuterated solvent, while ${ }^{31} \mathrm{P}$ chemical shifts are referred to external $85 \% \mathrm{H}_{3} \mathrm{PO}_{4}$. The IR spectra were recorded on a BioRad FTS 175 instrument. The following abbreviations were used: s, strong (IR); m, medium; s, singl et (NMR); d, doublet; $\mathrm{h}$, heptet; $\mathrm{m}$, multiplet. Microanalyses were carried out by the staff of the Microanalytical Service of the Department of Chemistry, National Cheng Kung University.

Synthesis of $\left[R u_{2}(C O)_{4}(\mu-S R)_{2}\left(P^{\prime}{ }_{3}\right)_{2}\right]\left(R^{\prime}=P h, R={ }^{t} B u\right.$ (3), 'Pr (4), Ph (5); $\mathbf{R}^{\prime}=\mathbf{M e}, \mathbf{R}=\mathbf{P h}$ (6)). To a solution of $\left[\mathrm{Ru}_{2}(\mathrm{CO})_{4}(\mathrm{MeCN})_{4}\left(\mathrm{PR}_{3}^{\prime}\right)_{2}\right]\left[\mathrm{BF}_{4}\right]_{2}{ }^{5 b}(0.26 \mathrm{mmol})$ dissolved in 20 $\mathrm{mL}$ of $\mathrm{MeCN}$ were added car efully deaerated HSR (1 mL) and $\mathrm{Et}_{3} \mathrm{~N}(2 \mathrm{~mL})$. The solution was stirred for $2 \mathrm{~h}$ at ambient temperature (ca. $28^{\circ} \mathrm{C}$ ), forming an orange-yel low precipitate for starting compounds with $\mathrm{R}^{\prime}=\mathrm{Ph}$ and solution for the compounds with $\mathrm{R}^{\prime}=\mathrm{Me}$. The solvent and volatiles were then removed under vacuum. Recrystallization from $\mathrm{CH}_{2} \mathrm{Cl}_{2} / \mathrm{MeOH}$ gave pure product.

3: Anal. Calcd for $\mathrm{C}_{48} \mathrm{H}_{48} \mathrm{O}_{4} \mathrm{P}_{2} \mathrm{Ru}_{2} \mathrm{~S}_{2}$ : C, 56.68; $\mathrm{H}, 4.75$. Found: C, 56.61; $\mathrm{H}, 4.75$. Yield: $87 \%$. IR $\left(\mathrm{CH}_{2} \mathrm{Cl}_{2}\right)$ : $\mathrm{v}_{\mathrm{CO}}, 2001$ $\mathrm{S}, 1965 \mathrm{~m}, 1931 \mathrm{~s} \mathrm{~cm}^{-1}$. ${ }^{1} \mathrm{H}$ NMR $\left(25{ }^{\circ} \mathrm{C}\right.$, acetone $\mathrm{d}_{6}, 400$ $\mathrm{MHz}): \delta 0.64(\mathrm{~s}, 18 \mathrm{H}), 7.53(\mathrm{~m}, 30 \mathrm{H}) .{ }^{31} \mathrm{P}\left\{{ }^{1} \mathrm{H}\right\} \mathrm{NMR}\left(25^{\circ} \mathrm{C}\right.$, $162 \mathrm{MHz}$ ): $\delta 27.21$ (s, $2 \mathrm{P}$ ) in acetone- $\mathrm{d}_{6}$ and $17.78(\mathrm{~s}, 2 \mathrm{P})$ in $\mathrm{CDCl}_{3}$

4: Anal. Calcd for $\mathrm{C}_{46} \mathrm{H}_{44} \mathrm{O}_{4} \mathrm{P}_{2} \mathrm{Ru}_{2} \mathrm{~S}_{2}$ : C, 55.86; $\mathrm{H}, 4.48$. Found: C, 55.68; $\mathrm{H}, 4.47$. Yield: $88 \%$. IR $\left(\mathrm{CH}_{2} \mathrm{Cl}_{2}\right): \mathrm{v}_{\mathrm{CO}}, 2010$ s, $2001 \mathrm{~s}, 1974 \mathrm{~m}, 1964 \mathrm{~m}, 1945 \mathrm{~s}, 1935 \mathrm{~s} \mathrm{~cm}^{-1}$. ${ }^{1} \mathrm{H}$ NMR (25 $\left.{ }^{\circ} \mathrm{C}, \mathrm{CDCl}_{3}, 400 \mathrm{MHz}\right): \delta 0.32(\mathrm{~d}, 12 \mathrm{H}, \mathrm{J}=6.6), 2.54(\mathrm{~h}, 2 \mathrm{H})$ for $\mathbf{4 A}$; and $\delta 0.31(\mathrm{~d}, 6 \mathrm{H}, \mathrm{J}=6.2), 1.03(\mathrm{~d}, 6 \mathrm{H}, \mathrm{J}=6.2), 2.39$ $(\mathrm{h}, 1 \mathrm{H}), 2.71(\mathrm{~h}, 1 \mathrm{H})$ for $\mathbf{4 B} .{ }^{31} \mathrm{P}\left\{{ }^{1} \mathrm{H}\right\}$ NMR $\left(25^{\circ} \mathrm{C}, \mathrm{CDCl}_{3}\right.$, $162 \mathrm{MHz}$ ): $\delta 19.97$ (s, $2 \mathrm{P}$ ) for 4A, and $\delta 26.54$ (s, $2 \mathrm{P}$ ) for 4B.

5: Anal. Calcd for $\mathrm{C}_{52} \mathrm{H}_{40} \mathrm{O}_{4} \mathrm{P}_{2} \mathrm{Ru}_{2} \mathrm{~S}_{2}$ : C, 59.08; $\mathrm{H}, 3.81$. Found: C, 59.15; $\mathrm{H}, 3.92$. Yield: $84 \%$. IR $\left(\mathrm{CH}_{2} \mathrm{Cl}_{2}\right): \mathrm{v}_{\mathrm{CO}}, 2016$ $\mathrm{s}, 1979 \mathrm{~m}, 1951 \mathrm{~s} \mathrm{~cm}^{-1}$. ${ }^{1} \mathrm{H} \mathrm{NMR}\left(25^{\circ} \mathrm{C}, \mathrm{CDCl}_{3}, 400 \mathrm{MHz}\right): \delta$ $6.68(\mathrm{~m}, 10 \mathrm{H}), 7.38(\mathrm{~m}, 30 \mathrm{H}) .{ }^{31} \mathrm{P}\left\{{ }^{1} \mathrm{H}\right\} \mathrm{NMR}\left(25^{\circ} \mathrm{C}, \mathrm{CDCl}_{3}\right.$, $162 \mathrm{MHz}$ ): $\delta 21.98(\mathrm{~s}, 2 \mathrm{P})$ for $\mathbf{5 A}$, and 26.15 (s, $2 \mathrm{P}$ ) for 5B.

6: $89 \%$ yield. Anal. Calcd for $\mathrm{C}_{16} \mathrm{H}_{32} \mathrm{O}_{4} \mathrm{P}_{2} \mathrm{Ru}_{2} \mathrm{~S}_{2}$ : C, 31.16; $\mathrm{H}$, 5.23. Found: $\mathrm{C}, 31.07 ; \mathrm{H}, 5.22$. IR $\left(\mathrm{CH}_{2} \mathrm{Cl}_{2}\right): \mathrm{v}_{\mathrm{CO}}, 2043 \mathrm{~s}$, $1983 \mathrm{~s}, 1970 \mathrm{sh}, 1937 \mathrm{~m} \mathrm{~cm}^{-1}$. ${ }^{1} \mathrm{H}$ NMR $\left(25^{\circ} \mathrm{C}\right.$, acetone-d $\mathrm{d}_{6}$, $400 \mathrm{MHz}): \delta 1.84(\mathrm{~d}, 9 \mathrm{H}, \mathrm{J}=10.1), 1.70(\mathrm{~d}, 9 \mathrm{H}, \mathrm{J}=10.1)$, $7.04(\mathrm{~m}, 10 \mathrm{H}) .{ }^{31 P}\left\{{ }^{1} \mathrm{H}\right\}$ NMR $\left(298\right.$ or $220 \mathrm{~K}$, acetone- $\mathrm{d}_{6}, 162$ $\mathrm{MHz}): \delta 6.42$ (s, $1 \mathrm{P}), 15.17$ (s, 1P).

Iodination of 3-6. To a solution of $\left[\mathrm{Ru}_{2}(\mathrm{CO})_{4}(\mu-\mathrm{SR})_{2-}\right.$ $\left.\left(\mathrm{PR}_{3}{ }_{3}\right)_{2}\right](0.12 \mathrm{mmol})$ dissolved in $20 \mathrm{~mL}$ of $\mathrm{CH}_{2} \mathrm{Cl}_{2}$ was added dropwise $4 \mathrm{~mL}$ (ca. $0.13 \mathrm{mmol}$ ) of an $\mathrm{I}_{2}$ solution prepared by dissolving $0.129 \mathrm{~g}(0.51 \mathrm{mmol})$ of $\mathrm{I}_{2}$ in $15 \mathrm{~mL}$ of $\mathrm{CH}_{2} \mathrm{Cl}_{2}$. The solution was stirred for $10 \mathrm{~min}$ at ambient temperature. The product, 7-10, was separated as a major yellow band by thinlayer chromatography (silica gel, $\mathrm{CH}_{2} \mathrm{Cl}_{2}$ :hexane $=1: 1$ ), using TLC plates (Kiesel guhr $60 \mathrm{~F}_{254}, \mathrm{E}$. Merck), and recrystallized from $\mathrm{CH}_{2} \mathrm{Cl}_{2} / \mathrm{MeOH}$.

[Ru $\mathbf{R}_{\mathbf{2}}(\mathbf{C O})_{\mathbf{4}}\left(\boldsymbol{\mu}-\mathbf{S t B u}_{\mathbf{2}} \mathbf{l}_{\mathbf{2}}\left(\mathbf{P P h}_{\mathbf{3}}\right)_{\mathbf{2}} \mathbf{\text { ] }}\right.$ (7): $\mathbf{7 7 \%}$ yield. Anal. Calcd for $\mathrm{C}_{48} \mathrm{H}_{48} \mathrm{I}_{2} \mathrm{O}_{4} \mathrm{P}_{2} \mathrm{Ru}_{2} \mathrm{~S}_{2}$ : C, 45.36; $\mathrm{H}, 3.81$. Found: C, 45.21; $\mathrm{H}, 3.83$. ${ }^{1} \mathrm{H}$ NMR $\left(25^{\circ} \mathrm{C}\right.$, acetone- $\left.\mathrm{d}_{6}, 400 \mathrm{MHz}\right): \delta 0.76(\mathrm{~s}, 18$ $\mathrm{H}), 7.73(\mathrm{~m}, 30 \mathrm{H})$. ${ }^{31 P}\left\{{ }^{1} \mathrm{H}\right\} N M R\left(300\right.$ or $220 \mathrm{~K}$, acetone- $\mathrm{d}_{6}$, $162 \mathrm{MHz}): \delta 44.58(\mathrm{~s}, 2 \mathrm{P})$. IR $\left(\mathrm{CH}_{2} \mathrm{Cl}_{2}\right): \mathrm{v}_{\mathrm{CO}}, 2057 \mathrm{~s}, 2049 \mathrm{~s}$, $2003 \mathrm{~s} \mathrm{~cm}^{-1}$. 
Table 1. Crystal Data

\begin{tabular}{|c|c|c|c|c|c|}
\hline $\begin{array}{l}\text { compd } \\
\text { formula } \\
\text { fw }\end{array}$ & $\begin{array}{l}3 \cdot \mathrm{CH}_{3} \mathrm{OH} \cdot 2 \mathrm{H}_{2} \mathrm{O} \\
\mathrm{C}_{49} \mathrm{H}_{48} \mathrm{O}_{7} \mathrm{P}_{2} \mathrm{Ru}_{2} \mathrm{~S}_{2} \\
1077.1\end{array}$ & $\begin{array}{l}\text { 4A } \\
\mathrm{C}_{46} \mathrm{H}_{44} \mathrm{O}_{4} \mathrm{P}_{2} \mathrm{Ru}_{2} \mathrm{~S}_{2} \\
988.9\end{array}$ & $\begin{array}{l}\text { 5B } \\
\mathrm{C}_{52} \mathrm{H}_{40} \mathrm{O}_{4} \mathrm{P}_{2} \mathrm{Ru}_{2} \mathrm{~S}_{2} \\
1057.8\end{array}$ & $\begin{array}{l}\mathbf{8} \\
\mathrm{C}_{22} \mathrm{H}_{28} \mathrm{O}_{4} \mathrm{P}_{2} \mathrm{Ru}_{2} \mathrm{~S}_{2} \\
684.67\end{array}$ & $\begin{array}{l}\mathbf{1 0} \\
\mathrm{C}_{22} \mathrm{H}_{28} \mathrm{I}_{2} \mathrm{O}_{4} \mathrm{P}_{2} \mathrm{Ru}_{2} \mathrm{~S}_{2} \\
988.9\end{array}$ \\
\hline color, habit & orange-yellow, chunk & yellow, rhombohedron & orange-yellow, chunk & yellow, prism & orange-yellow, equant \\
\hline diffractometer used & Siemens SMART-CCD & Siemens P4 & Nonius & Rigaku AF C7S & Siemens SMART-CCD \\
\hline $\begin{array}{l}\text { space group } \\
\mathrm{a}, \AA\end{array}$ & $\begin{array}{l}\text { monoclinic, C2/c } \\
48.905(2)\end{array}$ & $\begin{array}{l}\text { orthorhombic, } \mathrm{Cmc2}_{1} \\
24.522(4)\end{array}$ & $\begin{array}{l}\text { triclinic, P } \overline{1} \\
9.8356(14)\end{array}$ & $\begin{array}{l}\text { monoclinic, } \mathrm{P} 2{ }_{1} / \mathrm{n} \\
11.673(3)\end{array}$ & $\begin{array}{l}\text { monoclinic, } P 22_{1} / c \\
10.769(2)\end{array}$ \\
\hline $\mathrm{b}, \AA$ & $10.759(2)$ & $11.318(2)$ & $11.168(3)$ & $17.716(4)$ & $24.144(2)$ \\
\hline$c, \AA$ & $19.528(2)$ & $16.037(4)$ & $22.065(5)$ & $13.496(2)$ & $13.097(2)$ \\
\hline$\alpha$, deg & 90 & 90 & $82.16(3)$ & 90 & 90 \\
\hline$\beta$, deg & $97.97(2)$ & 90 & $78.92(3)$ & $94.59(2)$ & $112.56(2)$ \\
\hline$\gamma$, deg & 90 & 90 & $79.640(16)$ & 90 & 90 \\
\hline $\mathrm{V}, \AA^{3}$ & $10175(2)$ & $4451.1(14)$ & 2326.8(8) & $2782.0(10)$ & $3144.8(8)$ \\
\hline $\mathrm{Z}$ & 8 & 4 & 2 & 4 & 4 \\
\hline $\mathrm{D}_{\text {calcd }}, \mathrm{g} \mathrm{cm}^{-3}$ & 1.406 & 1.455 & 1.509 & 1.635 & 1.982 \\
\hline$\lambda(\mathrm{M} \circ \mathrm{K} \alpha), \AA$ & 0.71073 & 0.71073 & 0.7107 & 0.71069 & 0.71073 \\
\hline$F(000)$ & 4384 & 2208 & 1063 & 1368 & 1792 \\
\hline \multicolumn{6}{|l|}{ unit cell detn } \\
\hline no. $2 \theta$ range, deg & whole data & $25,14-28$ & $25,19-34$ & $25,15-23$ & whole data \\
\hline scan type & hemisphere & $\theta-\omega$ & $\theta-2 \theta$ & $\omega-2 \theta$ & hemisphere \\
\hline $2 \theta$ range, deg & $4-54$ & $3-50$ & $3-50$ & $6-47$ & $3-52$ \\
\hline $\mathrm{h}, \mathrm{k}, \mathrm{l}$ range & $\pm 49, \pm 12, \pm 23$ & $14,30,20$ & $\pm 18, \pm 19,26$ & $13,19, \pm 14$ & $\pm 13, \pm 28, \pm 15$ \\
\hline$\mu(\mathrm{M} \circ \mathrm{K} \alpha), \mathrm{cm}^{-1}$ & 7.85 & 8.84 & 4.75 & 13.76 & 31.83 \\
\hline $\begin{array}{l}\text { cryst size, mm } \\
\text { temp, K }\end{array}$ & $\begin{array}{l}0.48 \times 0.40 \times 0.35 \\
296\end{array}$ & $\begin{array}{l}0.5 \times 0.5 \times 0.5 \\
298\end{array}$ & $\begin{array}{l}0.50 \times 0.50 \times 0.60 \\
298\end{array}$ & $\begin{array}{l}0.25 \times 0.30 \times 0.33 \\
297\end{array}$ & $\begin{array}{l}0.52 \times 0.44 \times 0.36 \\
296\end{array}$ \\
\hline no. of measd rflns & 42104 & 4428 & 8198 & 4501 & 14136 \\
\hline no. of unique rflns & 8449 & 2098 & 8173 & 4269 & 5446 \\
\hline no. of obst rflns $\left(\mathrm{N}_{0}\right)$ & $7104(>3 \sigma)$ & $1660(>4 \sigma)$ & $6711(>2 \sigma)$ & $3400(>3 \sigma)$ & $4754(>3 \sigma)$ \\
\hline $\mathrm{R}^{\mathrm{a}}, \mathrm{R}_{\mathrm{w}}^{\mathrm{a}}$ & $0.038,0.043$ & $0.041,0.050$ & $0.026,0.030$ & $0.027,0.034$ & $0.034,0.042$ \\
\hline GOFa & 1.22 & 1.04 & 1.64 & 1.27 & 1.01 \\
\hline refinement program & SHELXTL-PLUS & SHELXTL-PLUS & NRCVAX & TEXSAN & SHELXTL-PLUS \\
\hline no. of ref params $\left(N_{p}\right)$ & 560 & 138 & 560 & 289 & 308 \\
\hline weighting scheme & {$\left[\sigma^{2}\left(\mathrm{~F}_{\mathrm{o}}\right)+0.0006 \mathrm{~F}_{\mathrm{o}}^{2}\right]^{-1}$} & {$\left[\sigma^{2}\left(\mathrm{~F}_{\mathrm{o}}\right)+0.0010 \mathrm{~F}_{\mathrm{o}}^{2}\right]^{-1}$} & {$\left[\sigma^{2}\left(\mathrm{~F}_{\mathrm{o}}\right)+0.0001 \mathrm{~F}_{0}^{2}\right]^{-1}$} & {$\left[\sigma^{2}\left(\mathrm{~F}_{\mathrm{o}}\right)\right]^{-1}$} & {$\left[\sigma^{2}\left(\mathrm{~F}_{\mathrm{o}}\right)+0.0009 \mathrm{~F}_{\mathrm{o}}^{2}\right]^{-1}$} \\
\hline second extinct coeff & 0 & 0 & $1.91(15) \times 10^{-4}$ & & \\
\hline$(\Delta \rho)_{\max }$, e $\AA^{-3}$ & 1.12 & 0.89 & 0.0065 & 0.32 & 0.97 \\
\hline$(\Delta \rho)_{\min }$, e $\AA^{-3}$ & -0.65 & -0.63 & -053 & -0.56 & -0.70 \\
\hline
\end{tabular}

[Ru $\mathbf{u}_{\mathbf{2}}(\mathbf{C O})_{\mathbf{4}}\left(\boldsymbol{\mu}-\mathbf{S}^{\mathrm{i}} \mathbf{P r}\right)_{\mathbf{2}} \mathbf{l}_{\mathbf{2}}\left(\mathbf{P P h}_{\mathbf{3}}\right)_{\mathbf{2}}$ ] (8): $83 \%$ yield. Anal. Calcd for $\mathrm{C}_{46} \mathrm{H}_{44} \mathrm{l}_{2} \mathrm{O}_{4} \mathrm{P}_{2} \mathrm{Ru}_{2} \mathrm{~S}_{2}$ : C, 44.45; $\mathrm{H}, 3.57$. Found: C, 44.21; $\mathrm{H}$, 3.60. ${ }^{1} \mathrm{H}$ NMR $\left(27^{\circ} \mathrm{C}\right.$, acetone $\left.\mathrm{d}_{6}, 400 \mathrm{MHz}\right): \delta 0.95(\mathrm{~d}, 12$ $\mathrm{H}, \mathrm{J}=6.7), 2.99(\mathrm{~h}, 2 \mathrm{H}), 7.60(\mathrm{~m}, 30 \mathrm{H}) .{ }^{31} \mathrm{P}\left\{{ }^{1} \mathrm{H}\right\} \mathrm{NMR}(300$ or $220 \mathrm{~K}$, acetone- $\left.\mathrm{d}_{6}, 162 \mathrm{MHz}\right): \delta 46.65(\mathrm{~s}, 2 \mathrm{P})$. IR $\left(\mathrm{CH}_{2^{-}}\right.$ $\mathrm{Cl}_{2}$ ): $\mathrm{v}_{\mathrm{CO}}, 2059 \mathrm{~s}, 2051 \mathrm{~s}, 2005 \mathrm{~s} \mathrm{~cm}^{-1}$.

[Ru $\mathbf{u}_{\mathbf{2}}(\mathbf{C O})_{\mathbf{4}}(\boldsymbol{\mu}-\mathbf{S P h})_{\mathbf{2}} \mathbf{I}_{\mathbf{2}}\left(\mathbf{P P h}_{\mathbf{3}}\right)_{\mathbf{2}}$ ] (9): $85 \%$ yield. Anal. Calcd for $\mathrm{C}_{52} \mathrm{H}_{40} \mathrm{l}_{2} \mathrm{O}_{4} \mathrm{P}_{2} \mathrm{Ru}_{2} \mathrm{~S}_{2}: \mathrm{C}, 47.64 ; \mathrm{H}, 3.08$. Found: $\mathrm{C}, 47.43$; $\mathrm{H}$, 3.11. ${ }^{1} \mathrm{H}$ NMR $\left(27^{\circ} \mathrm{C}\right.$, acetone- $\left.\mathrm{d}_{6}, 400 \mathrm{MHz}\right): \delta 7.47(\mathrm{~m}, 40$ $\mathrm{H}) .{ }^{31} \mathrm{P}\left\{{ }^{1} \mathrm{H}\right\} \mathrm{NMR}(300$ or $220 \mathrm{~K}$, acetone-d $6,162 \mathrm{MHz}$ ): $\delta$ 50.97 (s, $2 \mathrm{P})$. IR $\left(\mathrm{CH}_{2} \mathrm{Cl}_{2}\right): \mathrm{v}_{\mathrm{CO}}, 2070 \mathrm{sh}, 2062 \mathrm{~s}, 2017 \mathrm{~s} \mathrm{~cm}^{-1}$.

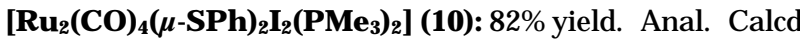
for $\mathrm{C}_{22} \mathrm{H}_{28} \mathrm{l}_{2} \mathrm{O}_{4} \mathrm{P}_{2} \mathrm{Ru}_{2} \mathrm{~S}_{2}$ : C, 28.16; $\mathrm{H}$, 3.01. Found: C, 28.11; $\mathrm{H}$, 3.01. ${ }^{1 \mathrm{H}} \mathrm{NMR}\left(27^{\circ} \mathrm{C}\right.$, acetone- $\left.\mathrm{d}_{6}, 400 \mathrm{MHz}\right): \delta 1.74(\mathrm{~d}, 9$ $\mathrm{H}, \mathrm{J}=10.8), 1.84(\mathrm{~d}, 9 \mathrm{H}, \mathrm{J}=10.8), 7.53(\mathrm{~m}, 10 \mathrm{H}) .{ }^{31} \mathrm{P}\left\{{ }^{1} \mathrm{H}\right\}$ NMR (300 or $220 \mathrm{~K}$, acetone-d, $162 \mathrm{MHz}$ ): $\delta 18.47$ (s, $1 \mathrm{P}$ ), 27.77 (s, 1 P). IR $\left(\mathrm{CH}_{2} \mathrm{Cl}_{2}\right): v_{\mathrm{CO}}, 2047 \mathrm{~s}, 2008 \mathrm{sh}, 1991 \mathrm{~s}, 1960$ $\mathrm{m} \mathrm{cm}^{-1}$.

Reaction of $\left[\mathrm{Ru}_{2}(\mathrm{CO})_{4}(\mu-S R)_{2} \mathbf{l}_{2}\left(\mathrm{PR}_{3}^{\prime}\right)_{2}\right]$ with $\mathbf{R}^{\prime \prime} \mathrm{SH}\left(\mathrm{R}^{\prime \prime}\right.$ $={ }^{\mathbf{B}} \mathbf{B},{ }^{\text {i }} \mathbf{P r}, \mathbf{P h}$ ) and $\mathbf{E} \mathbf{t}_{\mathbf{3}} \mathbf{N}$. (a) Reactions between $\mathbf{7}$ and R"SH/ $\mathrm{Et}_{3} \mathrm{~N}$ and that between $\mathbf{9}$ and ${ }^{\mathrm{t}} \mathrm{BuSH} / \mathrm{Et}_{3} \mathrm{~N}$ are quite similar, and only one typical example is shown below. Compound 7 (445 mg, $0.35 \mathrm{mmol}$ ) was dissolved in $20 \mathrm{~mL}$ of $\mathrm{MeCN}$, and to this solution were then added $1 \mathrm{~mL}$ of $\mathrm{PhSH}$ and $2 \mathrm{~mL}$ of $E t_{3} \mathrm{~N}$. The solution was stirred for $2 \mathrm{~h}$, forming a orange-yellow precipitate. The solvent and volatiles were then removed under vacuum, and the residue was taken up in a minimum amount of $\mathrm{CH}_{2} \mathrm{Cl}_{2}$. The products were separated by thin-layer chromatography using $\mathrm{CH}_{2} \mathrm{Cl}_{2}$ /hexane mixed solvents to give $63 \mathrm{mg}$ of PhSSPh (83\%) and $274 \mathrm{mg}$ of $\mathbf{3}$ (77\%). (b) Reaction between 9 and $\mathrm{PhSH} / \mathrm{Et}_{3} \mathrm{~N}$ : Compound 9 (203 mg, $0.155 \mathrm{mmol}$ ) was dissolved in $10 \mathrm{~mL}$ of $\mathrm{MeCN}$ to form a clear yellow solution, and to this solution were then added $1 \mathrm{~mL}$ of $\mathrm{Et}_{3} \mathrm{~N}$ and $0.5 \mathrm{~mL}$ of $\mathrm{PhSH}$. Upon addition of $\mathrm{PhSH}$, the orangeyellow color developed immediately and an orange-yellow precipitate appeared within $3 \mathrm{~min}$. The suspension was stirred for an additional $30 \mathrm{~min}$. The precipitate was collected, washed with $5 \mathrm{~mL}$ of $\mathrm{MeOH}$ three times, and dried under vacuum to afford $148 \mathrm{mg}$ of the product $\left[\mathrm{Ru}_{2}(\mathrm{CO})_{4}(u-\mathrm{SPh})_{2-}\right.$ $\left.(\mathrm{SPh})_{2}\left(\mathrm{PPh}_{3}\right)_{2}\right]$ (11) in $75 \%$ yield. Anal. Calcd for $\mathrm{C}_{64} \mathrm{H}_{50} \mathrm{O}_{4} \mathrm{P}_{2} \mathrm{Ru}_{2} \mathrm{~S}_{4}$ : C, 60.27; $\mathrm{H}, 3.95$. Found: $\mathrm{C}, 60.17 ; \mathrm{H}, 3.95$. IR $\left(\mathrm{CH}_{2} \mathrm{Cl}_{2}\right): \mathrm{V}_{\mathrm{CO}}, 2045 \mathrm{~s}, 2032 \mathrm{sh}, 1985 \mathrm{~s} \mathrm{~cm}^{-1}$. ${ }^{1} \mathrm{H}$ NMR (27 $\left.{ }^{\circ} \mathrm{C}, \mathrm{CDCl}_{3}, 400 \mathrm{MHz}\right): \delta 7.39(\mathrm{~m}, 50 \mathrm{H}) .{ }^{31} \mathrm{P}\left\{{ }^{1} \mathrm{H}\right\} \mathrm{NMR}(300$ $\left.\mathrm{K}, \mathrm{CDCl}_{3}, 162 \mathrm{MHz}\right): \delta 19.47(\mathrm{~s}, 2 \mathrm{P})$.

Single-Crystal X-ray Diffraction Studies of 3, 4A, 5B, 6, and 10. Suitable single crystals were grown from $\mathrm{CH}_{2} \mathrm{Cl}_{2} /$ $\mathrm{MeOH}$ or $\mathrm{CH}_{2} \mathrm{Cl}_{2}$ /hexane at room temperature and chosen for single crystal structure determinations. The X-ray diffraction data of 4A, 5B, and 6 were measured on a four-circle diffractometer, and those of $\mathbf{3}$ and $\mathbf{1 0}$ were measured in frames with increasing $\omega$ ( $0.3 \mathrm{deg} /$ frame) and with the scan speed at 10.00 s/frame on a Siemens SMART-CCD instrument, equipped with a normal focus and $3 \mathrm{~kW}$ sealed-tube X-ray source. For data collected on the four-circle diffractometer, three standard reflections were monitored every hour or every 50 reflections throughout the collection. The variation was less than $2 \%$. Empirical absorption corrections were carried out based on an azimuthal scan. For $\mathbf{6}$, the structure was solved by direct methods and refined by a full-matrix least-squares procedure using TEXSAN. ${ }^{7}$ For $\mathbf{5 B}$, the structures were solved by the heavy-atom method and refined by a full-matrix least-squares procedure using NRCVAX. ${ }^{8}$ F or $\mathbf{3}, \mathbf{4 A}$, and 10, the structures were solved by direct methods and refined by a full-matrix least-squares procedure using SHELXTL-PLUS. ${ }^{9}$ Neutral atom scattering factors for non-hydrogen atoms and the values for $\Delta f^{\prime}$ and $\Delta f^{\prime \prime}$ described in each software ${ }^{7-9}$ were used. The other essential details of single-crystal data measurement and refinement are listed in Table 1 . One molecule of $\mathrm{MeOH}$ and two molecules of $\mathrm{H}_{2} \mathrm{O}$ were found in the asymmetric unit of

(7) Crystal Structure Analysis Package, Molecular Structure Corporation, Texas, 1985 and 1992.

(8) Gabe, E. J .; Le page, Y.; Charland, J .-P.; Lee, F. L.; White, P. S. J. Appl. Crystallogr. 1989, 22, 384.

(9) (a) Sheldrick, G. M. SHELXTL-Plus Crystallographic System, release 4.21; Siemens Analytical X-ray Instruments: Madison, WI, 1991. (b) Siemens Analytical X-ray Instruments Inc.: Karlsruhe Germany, 1991. 


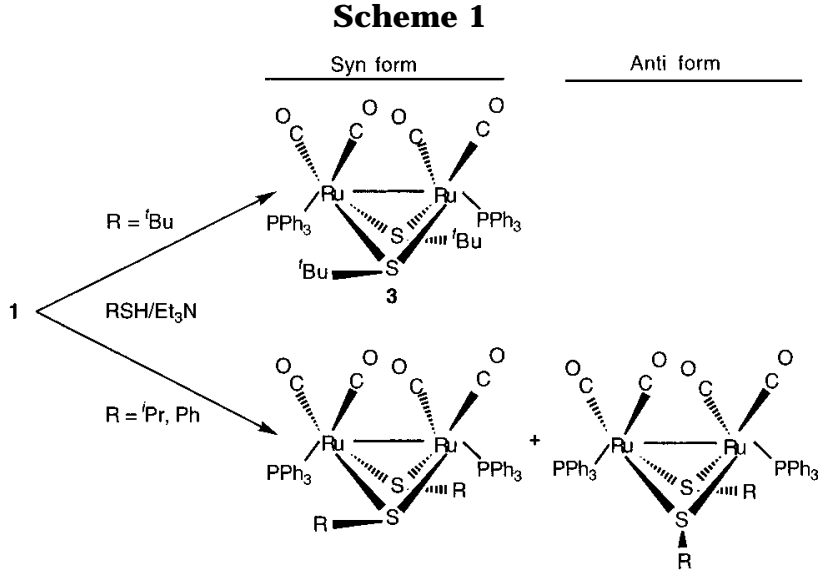

$\left(\mathrm{R}={ }^{i} \operatorname{Pr}(\mathbf{4 A}), \operatorname{Ph}(5 \mathrm{~A})\right) \Longrightarrow\left(\mathrm{R}={ }^{i} \operatorname{Pr}(\mathbf{4 B}), \operatorname{Ph}(\mathbf{5 B})\right)$
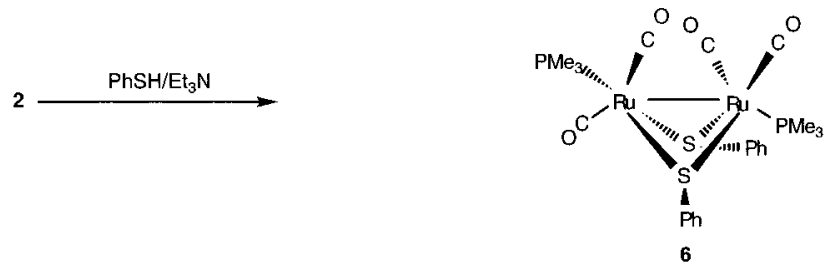

the crystals used for 3. The solvent hydrogen positions in this structure were not included in the structure refinement.

\section{Results and Discussion}

Synthesis, Structures, and Solution Behavior of $\left[R u_{2}(C O)_{4}(\mu-S R)_{2}\left(P^{\prime}{ }_{3}\right)_{2}\right]$ (3-6). Following one of our recent reports that the acetonitrile ligands of $\mathbf{1}$ and $\mathbf{2}$ can be easily replaced by weak anions such as $\mathrm{NO}_{3}{ }^{-}, 10$ we rediscovered that the temperature for reaction between 1 and 1,2-benzenedithiol to produce $\left[\mathrm{Ru}_{2}(\mathrm{CO})_{4-}\right.$ $\left.\left(\mu, \eta^{2}-\mathrm{S}_{2} \mathrm{C}_{6} \mathrm{H}_{4}\right)\left(\mathrm{PPh}_{3}\right)_{2}\right]^{5 \mathrm{~b}}$ can be reduced to the ambient temperature. Likewise the reaction of $\mathbf{1}$ and $\mathbf{2}$ with a thiol in the presence of a base such as $\mathrm{Et}_{3} \mathrm{~N}$ can occur readily at ambient temperature to give 3-6 in satisfactory yield (Scheme 1). The diruthenium trimethylphosphine derivative was found to be more air-sensitive than the triphenylphosphine analogue. Both the thiol and $\mathrm{Et}_{3} \mathrm{~N}$ should be carefully deaerated before addition to compounds $\mathbf{1}$ and $\mathbf{2}$, especially for the preparation of $\mathbf{6}$. This compound is orange-yellow. However, when aerated thiol and $\mathrm{Et}_{3} \mathrm{~N}$ were used to react with 2, the resulted orangered to red products contained obviously multiple components displaying more than $15^{31} \mathrm{P}\left\{{ }^{1} \mathrm{H}\right\}$ NMR singlets between $\delta-20$ and 33 in $\mathrm{CDCl}_{3}$.

Compound $\mathbf{3}$ was previously reported to be formed either from the thermal degradation of $\left[\mathrm{Ru}_{3}(\mathrm{CO})_{9}\left(\mathrm{PPh}_{3}\right)_{3}\right]$ with ${ }^{\mathrm{B}} \mathrm{B}$ SH by Cabeza et al. in $1989^{5 \mathrm{a}}$ or from a long $(20 \mathrm{~h})$ thermal displacement reaction between $\left[\mathrm{Ru}_{2}(\mathrm{CO})_{4}\left(\mu-\mathrm{O}_{2} \mathrm{CH}\right)_{2}\left(\mathrm{PPh}_{3}\right)_{2}\right]$ and the thiol in refluxing toluene (bp $110^{\circ} \mathrm{C}$ ) by Ros et al. in 1995. ${ }^{5 \mathrm{c}}$ Although it is not unreasonable that both syn and anti isomers are produced under these reaction conditions, the ${ }^{31} \mathrm{P}\left\{{ }^{1} \mathrm{H}\right\}$ chemical shifts of Ros's two isomers are rather high at $\delta 45.3$ for syn and 39.5 for anti, compared with the values of 23.87 reported for Cabeza's compound $5 \mathrm{a}$ and 17.78 measured for $\mathbf{3}$ in our laboratory. The ${ }^{31} \mathrm{P}\left\{{ }^{1} \mathrm{H}\right\}$ singlet observed for this compound remains at $220 \mathrm{~K}$

(10) Shiu, K.-B.; Yang, L.-T.; J ean, S.-W.; Li, C.-H.; Wu, R.-L.; Wang, .-C.; Liou, L.-S.; Chiang, M. Y. Inorg. Chem. 1996, 35, 7845.

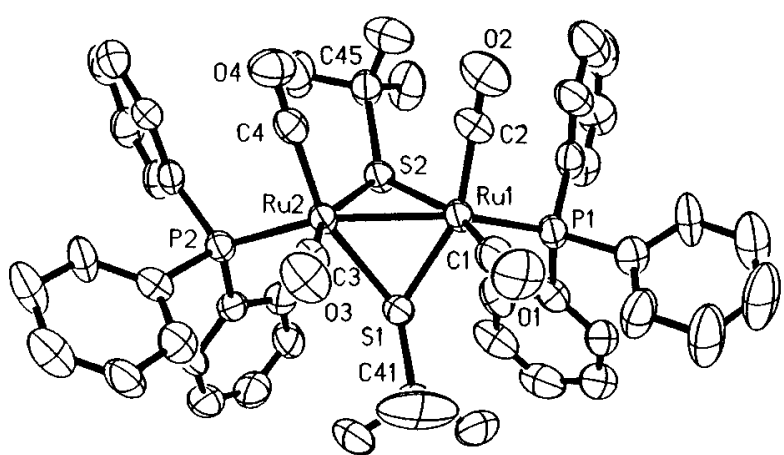

Figure 1. ORTEP plot of $\left[\mathrm{Ru}_{2}(\mathrm{CO})_{4}(\mu-\mathrm{StBu})_{2}\left(\mathrm{PPh}_{3}\right)_{2}\right](3)$. Selected bond distances $(\AA)$ : $R u(1)-R u(2)=2.640(1), R u-$ $(1)-S(1)=2.319(1), R u(1)-S(2)=2.422(1), R u(1)-P(1)=$ 2.449(1), $\mathrm{Ru}(1)-\mathrm{C}(1)=1.867(4), \mathrm{Ru}(1)-\mathrm{C}(2)=1.867(5)$, $\mathrm{Ru}(2)-\mathrm{S}(1)=2.564(1), \mathrm{Ru}(2)-\mathrm{S}(2)=2.466(1), \mathrm{Ru}(2)-\mathrm{P}(2)$ $=2.265(1), \mathrm{Ru}(2)-\mathrm{C}(3)=1.887(4), \mathrm{Ru}(2)-\mathrm{C}(4)=1.945(5)$. Selected bond angles (deg): $\mathrm{Ru}(1)-\mathrm{Ru}(2)-\mathrm{S}(1)=52.9(1)$ $\mathrm{Ru}(1)-\mathrm{Ru}(2)-\mathrm{S}(2)=56.5(1), \mathrm{Ru}(1)-\mathrm{Ru}(2)-\mathrm{P}(2)=148.6-$ (1), $\mathrm{Ru}(2)-\mathrm{Ru}(1)-\mathrm{S}(1)=61.9(1), \mathrm{Ru}(2)-\mathrm{Ru}(1)-\mathrm{S}(2)=58.1-$ (1), Ru(2)-Ru(1)-P(1) = 150.3(1), Ru(1)-S(1)-Ru(2) = 65.2(1), $\mathrm{Ru}(1)-\mathrm{S}(2)-\mathrm{Ru}(2)=65.4(1), \mathrm{P}(1)-\mathrm{Ru}(1)-\mathrm{S}(1)=$ 96.6(1), $P(1)-R u(1)-S(2)=97.9(1), P(2)-R u(2)-S(1)=$ 100.8(1), $\mathrm{P}(2)-\mathrm{Ru}(2)-\mathrm{S}(2)=100.6(1)$.

in acetone- $d_{6}$, or at $420 \mathrm{~K}$ in acetophenone- $\mathrm{d}_{3}$, indicating probably the presence of only one isomer at either high or low temperature. On the basis of the three-carbonylstretching-band pattern observed for $\mathbf{3}$ dissolved in $\mathrm{CH}_{2-}$ $\mathrm{Cl}_{2}$, the structure is not in the anti form $\left(\mathrm{C}_{\mathrm{s}}\right.$ symmetry), but in the syn geometry $\left(\mathrm{C}_{2 v}\right.$ symmetry), which was confirmed by X-ray diffraction methods (Figure 1 ). The high-temperature ${ }^{31 P}$ NMR evidence reflects that the syn geometry is more stable thermodynamically than the anti form, if this form can be prepared in some way. It is hence quite surprising that Ros's isolated "syn" isomer was found to be a minor product with "syn"/"anti" $=1: 8^{5 \mathrm{c}}$

The isolated $\mathbf{4}$ and $\mathbf{5}$ exist in solution as equilibrium mixtures of syn (isomer A) and anti (isomer B) isomers, as evidenced by the following: (1) similar NMR and IR spectra were obtained by dissolving either the products $\mathbf{4}$ and $\mathbf{5}$ or their single crystals (4A and 5B); (2) the syn: anti ratio was found to be changeless within experimental error, even after a toluene solution of 4 (or 5) was heated under reflux for more than $80 \mathrm{~h}$; and (3) two exchange cross peaks, indicating clearly $\mathrm{P}_{\text {syn }} \leftrightarrow \mathrm{P}_{\text {anti, }}$, were shown in the ${ }^{11} \mathrm{P}\left\{{ }^{1} \mathrm{H}\right\}$ NOESY NMR experiments ${ }^{11}$ for 4 and 5 in $\mathrm{CDCl}_{3}$ at $298 \mathrm{~K}$. The spectral data support that 4A (the syn form) is the major isomer for 4, whereas 5B (the anti form) is the major component for $\mathbf{5}$. The observed syn/anti (or 4A:4B) integration ratio of 1.16:1 based on the ${ }^{1} \mathrm{H}$ NMR doublets assigned for the methyl groups of i $\mathrm{Pr}$ of $\mathbf{4}$ is close to that of 1.14:1 based on the inverse-gated ${ }^{31} \mathrm{P}\left\{{ }^{1} \mathrm{H}\right\}$ NMR singlets (i.e., without NOE) in $\mathrm{CDCl}_{3}$. Similarly, the calculated integration ratio between one carbonyl ${ }^{13} \mathrm{C}$ NMR singlet for 5A (the syn form) and two carbonyl singl ets for 5B (the anti form) is 1.14:1:1, based on the observed $\mathbf{5 A}$ : 5B integration ratio of 1:1.766 found as two inversegated ${ }^{1} \mathrm{P}\left\{{ }^{1} \mathrm{H}\right\}$ NMR singlets, is close to the observed integration ratio of 1.13:1:1 based on the three broad

(11) Bodenhausen, G.; Ernst, R. R. J . Am. Chem. Soc. 1982, 104, 1304. 


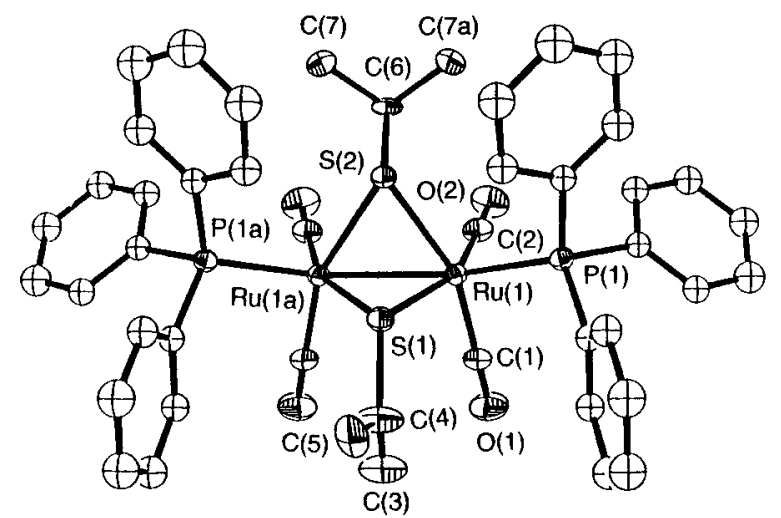

Figure 2. ORTE P plot of $\left[\mathrm{Ru}_{2}(\mathrm{CO})_{4}\left(\mu-\mathrm{SiPr}_{2}\left(\mathrm{PPh}_{3}\right)_{2}\right](\mathbf{4 A})\right.$. Selected bond distances $(\AA)$ : $R u(1)-R u(1 a)=2.694(1)$, Ru$(1)-S(1)=2.424(3), R u(1)-S(2)=2.422(3), R u(1)-P(1)=$ 2.375(3), $\mathrm{Ru}(1)-\mathrm{C}(1)=1.874(10), \mathrm{Ru}(1)-\mathrm{C}(2)=1.855(10)$. Selected bond angles (deg): Ru(1a)-Ru(1)-S(1) = 56.3(1), Ru(1a)-Ru(1)-S(2) = 56.2(1), Ru(1a)-Ru(1)-P(1) = 148.7(1), Ru(1)-S(1)-Ru(1a) = 67.5(1), P(1)-Ru(1)-S(1) $=97.5(1), P(1)-R u(1)-S(2)=102.6(1)$.

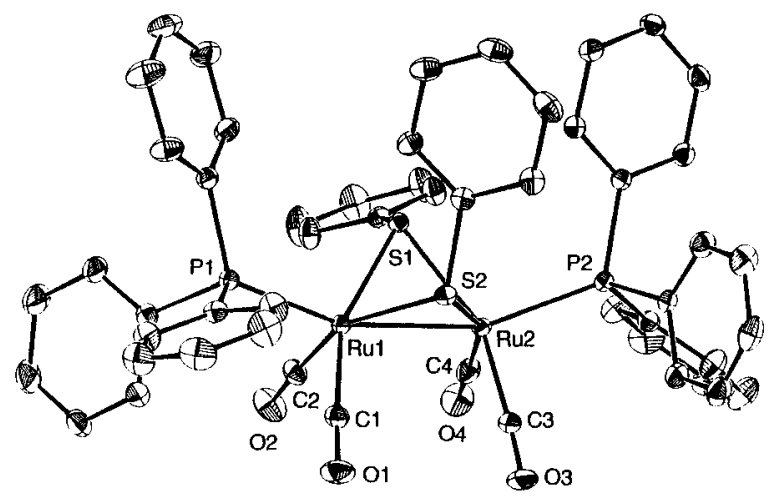

Figure 3. ORTEP plot of $\left[\mathrm{Ru}_{2}(\mathrm{CO})_{4}(u-\mathrm{SPh})_{2}\left(\mathrm{PPh}_{3}\right)_{2}\right](5 \mathrm{~B})$. Selected bond distances $(\AA): \operatorname{Ru}(1)-R u(2)=2.6788(10)$, $\mathrm{Ru}(1)-\mathrm{S}(1)=2.4285(9), \mathrm{Ru}(1)-\mathrm{S}(2)=2.4358(11), \mathrm{Ru}(1)-$ $\mathrm{P}(1)=2.3897(11), \mathrm{Ru}(1)-\mathrm{C}(1)=1.868(3), \mathrm{Ru}(1)-\mathrm{C}(2)=$ $1.861(3), \mathrm{Ru}(2)-\mathrm{S}(1)=2.4260(8), \mathrm{Ru}(2)-\mathrm{S}(2)=2.4323(9)$, $\mathrm{Ru}(2)-\mathrm{P}(2)=2.3762(11), \mathrm{Ru}(2)-\mathrm{C}(3)=1.874(3), \mathrm{Ru}(2)-$ $C(4)=1.870(3)$. Selected bond angles (deg): Ru(2)-Ru$(1)-S(1)=51.463(24), R u(2)-R u(1)-S(2)=56.55(3), R u-$ (2) $-\mathrm{Ru}(1)-\mathrm{P}(1)=159.917(24), \mathrm{Ru}(1)-\mathrm{Ru}(2)-\mathrm{S}(1)=56.55(3)$, $\mathrm{Ru}(1)-\mathrm{Ru}(2)-\mathrm{S}(2)=56.68(3), \mathrm{Ru}(1)-\mathrm{Ru}(2)-\mathrm{P}(2)=156.929$ (24), $R u(1)-S(1)-R u(2)=66.98(3), R u(1)-S(2)-R u(2)=$ 66.77(3), $\mathrm{P}(1)-\mathrm{Ru}(1)-\mathrm{S}(1)=109.04(4), \mathrm{P}(1)-\mathrm{Ru}(1)-\mathrm{S}(2)$ $=109.96(4), P(2)-R u(2)-S(1)=106.57(4), P(2)-R u(2)-$ $S(2)=107.81(4)$.

${ }^{13} \mathrm{C}$ NMR carbonyl singlets measured in $\mathrm{CDCl}_{3}$. Both the ${ }^{31} \mathrm{P}\left\{{ }^{1} \mathrm{H}\right\}$ NMR singlet and the ${ }^{13} \mathrm{C} N \mathrm{NMR}$ carbonyl singlet for the syn form were observed at an upfield position relative to those for the anti form $\left({ }^{31} \mathrm{P}\left\{{ }^{1} \mathrm{H}\right\}\right.$ NMR $\delta 19.97$ for $\mathbf{4 A}$ vs 26.54 for $\mathbf{4 B}$ and 21.98 for $\mathbf{5 A}$ vs. 26.15 for $5 B ;{ }^{13} \mathrm{C}\left\{{ }^{1} \mathrm{H}\right\}$ NMR 202.0 for $\mathbf{5 A}$ vs 203.4 and 204.6 for 5B). The single-crystal structures were determined by X-ray diffraction methods, confirming the stereochemistry with the syn form for $\mathbf{4 A}$ (Figure 2) and the anti form for 5B (Figure 3). Quite coincidentally, the major isomer is the one giving the structures. The spectral and the X-ray crystal structural evidence apparently explain why the two isomers of $\mathbf{4}$ and $\mathbf{5}$ cannot be separated in our hands by either silica or alumina column chromatography by $\mathrm{CH}_{2} \mathrm{Cl}_{2}$ /hexane mixtures, although such a separation method was previously reported in obtaining one "syn" and two "anti" isomers of a similar compound, $\left[\mathrm{Ru}_{2}(\mathrm{CO})_{4}(\mu-\mathrm{SBz})_{2-}\right.$ $\left.\left(\mathrm{PPh}_{3}\right)_{2}\right](\mathrm{Bz}=$ benzyl $)$, by Ros's group. ${ }^{5 \mathrm{c}}$ The syn-anti equilibria of the organothio-bridged deriviatives of iron carbonyl, $\left[\mathrm{Fe}_{2}(\mathrm{CO})_{6}(u-\mathrm{SR})_{2}\right]$ (with $\mathrm{R}=\mathrm{Me}, \mathrm{Et}$ ), and of their monosubstitution products, $\left[\mathrm{Fe}_{2}(\mathrm{CO})_{5}(\mu-\mathrm{SMe})_{2}\left(\mathrm{P}^{\mathrm{n}}\right.\right.$ $\left.\mathrm{Bu}_{3}\right)$ ], were reported previously to have the predominant form in anti geometry for the former but in syn geometry for the latter.4e The disubstitution products of a related selenium compound, $\left[\mathrm{Ru}_{2}(\mathrm{CO})_{6}(\mu-\mathrm{SePh})_{2}\right]$, with $\mathrm{PPh}_{3}$ were al so found to consist of both forms, with the anti mode favored. ${ }^{6}$ Apparently both steric and electronic factors should be combined to account for the different ratio between the two forms. ${ }^{4 g, h}$

Dinuclear thiolato-bridged phosphine carbonyl complexes $\left[\mathrm{M}_{2}(\mathrm{CO})_{4}\left(u-\mathrm{SR}^{\prime}\right)_{2}\left(\mathrm{PR}_{3}\right)_{2}\right](\mathrm{M}=\mathrm{Fe}, \mathrm{Ru})$ were rarely characterized by $\mathrm{X}$-ray diffraction methods and limited in the syn form of $\left[\mathrm{Fe}_{2}(\mathrm{CO})_{4}(u \text {-SMe })_{2}\left(\mathrm{PMe}_{3}\right)_{2}\right]$ in $1977,4 \mathrm{i}$ and no crystal structures containing diruthenium analogues have been characterized so far. Hence, 3, 4A, and 5B are the first crystallographically characterized syn and anti structures of diruthenium thiol ato-bridged carbonyl phosphine complexes. The Ru-Ru distances of 2.640(1) $\AA$ in 3, 2.694(1) $\AA$ in 4A, and 2.679(1) $\AA$ in 5B are similar to each other and fall within the range $2.558-2.873 \AA$ of other singly $\mathrm{Ru}-\mathrm{Ru}$ bonded complexes with ligation of $\mathrm{PPh}_{3}$ ligands. ${ }^{10,12}$ Structure $\mathbf{4 A}$ has a crystallographically imposed mirror plane containing the midpoint of the Ru-Ru bond, two sulfur atoms, and four carbon atoms, $C(3)-C(6)$ (Figure 2). Like the previously reported structure with two thiolato bridges, $\left[\mathrm{Fe}_{2}(\mathrm{CO})_{4}(\mu-\mathrm{SMe})_{2}\left(\mathrm{PMe}_{3}\right)_{2}\right]_{,}{ }^{4 i}$ and those with one dithiol ato bridge, $\left[\mathrm{Ru}_{2}(\mathrm{CO})_{4}\left(\mu, \eta^{2}-\mathrm{S}_{2}\left(\mathrm{C}_{6} \mathrm{H}_{4}\right)\left(\mathrm{PPh}_{3}\right)_{2}\right]^{5 \mathrm{~b}}\right.$ and $\left[\mathrm{Ru}_{2}-\right.$ $\left.(\mathrm{CO})_{4}\left(\mu, \eta^{2}-\mathrm{S}_{2}\left(\mathrm{CH}_{2}\right)_{3}\right)\left(\mathrm{PPh}_{3}\right)_{2}\right],{ }^{, \mathrm{c}}$ the three thiolato-bridged structures $\mathbf{3}$ (Figure 1 ), 4A (Figure 2 ), and 5B (Figure 3) follow to have a common structure type with the two thiolato bridges all being located cis to the two phosphine groups.

Like 4 and 5, product 6 exhibited two ${ }^{31} \mathrm{P}\left\{{ }^{1} \mathrm{H}\right\}$ NMR singlets at $\delta 6.42$ and 15.17 at ambient temperature $(298 \mathrm{~K})$ and at a low temperature $(220 \mathrm{~K})$. However, the single-crystal X-ray structure evidence of $\mathbf{6}$ did not support the presence of both syn and anti isomers in solution, but revealed an unprecedented structure type for only one anti isomer. The two thiolato bridges are located cis to one but trans to the other phosphine ligand, resulting in two inequivalent phosphine atoms and two ${ }^{31} \mathrm{P}\left\{{ }^{1} \mathrm{H}\right\}$ NMR singlets in the NMR spectra, with $\mathrm{d}(\mathrm{Ru}-\mathrm{Ru})=2.6923(8) \AA$ in 6 (Figure 4). The four-band $\mathrm{v}_{\mathrm{CO}}$ pattern displayed by a $\mathrm{CH}_{2} \mathrm{Cl}_{2}$ solution of $\mathbf{6}$ in an IR spectrum is consistent with the $C_{1}$ symmetry of this structure. However, the $v_{c o}$ pattern should not be overemphasized without consideration of other evidence such as NMR results in the characterization of the related structures, for the bands may be overlapped into a different pattern or may not be resolved by the IR instrument, explaining a six-band and a three-band pattern observed for $\mathbf{4}$ and 5, respectively, each containing an equilibrium mixture of two differently weighted

(12) (a) Sherlock, S. J .; Cowie, M.; Singleton, E.; Steyn, M. M. de V J. Organomet. Chem. 1989, 361, 353. (b) Garcia-Granda, S.; ObesoRosete, R.; Gonzalez, J . M. R.; Anillo, A. Acta Crystallogr. 1990, C46, 2043. (c) Shiu, K.-B.; Peng, S.-M.; Cheng, M.-C. J . Organomet. Chem. 1993, 452, 143. (d) Klemperer, W. G.; Bianxia, Z. Inorg. Chem. 1993, 32,5821 


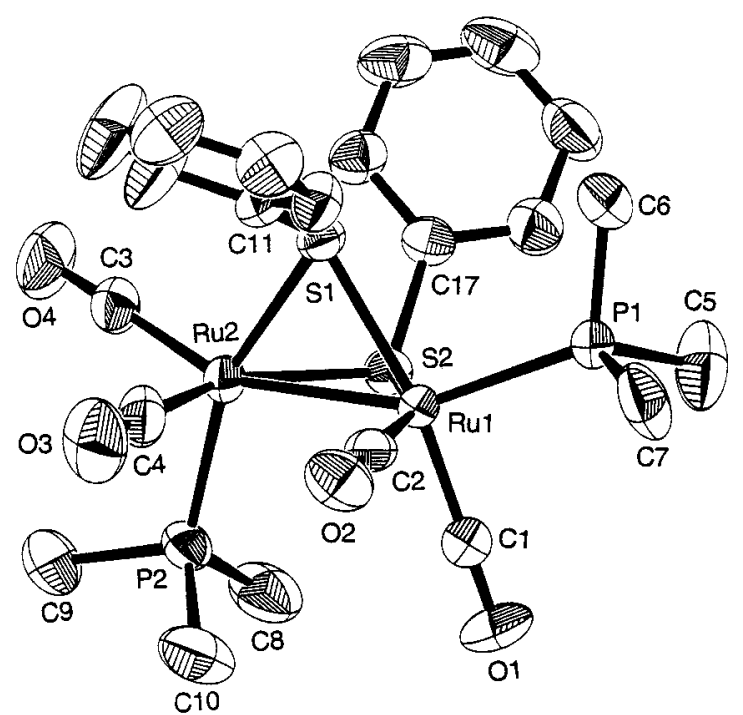

Figure 4. ORTEP plot of $\left[\mathrm{Ru}_{2}(\mathrm{CO})_{4}(\mu-\mathrm{SPh})_{2}\left(\mathrm{PMe}_{3}\right)_{2}\right](\mathbf{6})$. Selected bond distances: $\mathrm{Ru}(1)-\mathrm{Ru}(2)=2.6923(8), \mathrm{Ru}(1)-$ $\mathrm{S}(1)=2.423(1), \mathrm{Ru}(1)-\mathrm{S}(2)=2.433(1), \mathrm{Ru}(1)-\mathrm{P}(1)=2.357-$ (1), $\mathrm{Ru}(1)-\mathrm{C}(1)=1.862(4), \mathrm{Ru}(1)-\mathrm{C}(2)=1.868(5), \mathrm{Ru}(2)-$ $\mathrm{S}(1)=2.417(1), \mathrm{Ru}(2)-\mathrm{S}(2)=2.407(1), \mathrm{Ru}(2)-\mathrm{P}(2)=$ $2.327(1), R u(2)-C(3)=1.891(4), R u(2)-C(4)=1.880(5) \AA$. Selected bond angles: $R u(2)-R u(1)-S(1)=56.09(3)$, Ru(2) $-\mathrm{Ru}(1)-\mathrm{S}(1)=55.76(3), \mathrm{Ru}(2)-\mathrm{Ru}(1)-\mathrm{P}(1)=151.95-$ (3), $\mathrm{Ru}(1)-\mathrm{Ru}(2)-\mathrm{S}(1)=56.32(3), \mathrm{Ru}(1)-\mathrm{Ru}(2)-\mathrm{S}(2)=$ 56.65(3), Ru(1)-Ru(2)-P(2) = 102.74(3), Ru(1)-S(1)-Ru$(2)=67.60(3), R u(1)-S(2)-R u(2)=67.60(3), P(1)-R u(1)-$ $\mathrm{S}(1)=99.71(4), \mathrm{P}(1)-\mathrm{Ru}(1)-\mathrm{S}(2)=109.13(4), \mathrm{P}(2)-\mathrm{Ru}(2)-$ $\mathrm{S}(1)=159.04(4), \mathrm{P}(2)-\mathrm{Ru}(2)-\mathrm{S}(2)=90.81(4)^{\circ}$.

Scheme 2

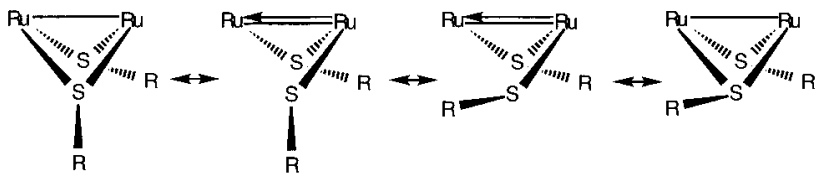

syn and anti isomers. A mechanism probably involving conversion of a bridging into a terminal thiolato group with concomitant formation of multiple Ru-Ru bonding interactions is proposed to account for the equilibrium process between the syn and anti forms of $\mathbf{4}$ and $\mathbf{5}$ in solution (Scheme 2).

Synthesis, Structures, and Reactions of $\left[R \mathbf{u}_{2}-\right.$ $(\mathbf{C O})_{\mathbf{4}}\left(\boldsymbol{\mu}-\mathbf{S R}^{\prime}\right)_{\mathbf{2}} \mathbf{I}_{\mathbf{2}}\left(\mathbf{P R}_{\mathbf{3}}\right)_{\mathbf{2}} \mathbf{]} \mathbf{( 7 - 1 0 )}$. The electrophilic addition of $\mathrm{I}_{2}$ to $\mathrm{CH}_{2} \mathrm{Cl}_{2}$ solutions of $\mathbf{3} \mathbf{- 6}$ generates al most immediately at ambient temperature the diiodide adducts $\left[\mathrm{Ru}_{2}(\mathrm{CO})_{4}\left(\mu-\mathrm{SR}^{\prime}\right)_{2} \mathrm{I}_{2}\left(\mathrm{PR}_{3}\right)_{2}\right](\mathbf{7}-\mathbf{1 0})$ (Scheme 3). It appears that there are two different classes of products, as shown in the three-band and the four-band $\mathrm{v}_{\mathrm{CO}}$ patterns observed for 7-9 and 10, respectively. Quite coincidentally, the difference between the highest $v_{C O}$ bands of 7-10 and their respective precursor compounds 3-6 is as high as about $50 \mathrm{~cm}^{-1}$ for $7-9$ and as low as $4 \mathrm{~cm}^{-1}$ for $\mathbf{1 0}$ (2057 in 7 vs 2001 in 3; 2059 in 8 vs 2001 in 4; 2070 in 9 vs 2016 in 4; 2047 in 10 vs 2043 $\mathrm{cm}^{-1}$ in 6). The single crystals of $\mathbf{1 0}$ were easily grown from $\mathrm{CH}_{2} \mathrm{Cl}_{2} /$ hexane at ambient temperature, and the structure was soon found to keep the unique structure just like the precursor 6 but change the geometry from anti in $\mathbf{6}$ into syn in $\mathbf{1 0}$ (Figure 5). This novel structure with inequivalent phosphine atoms remains not only in the solid state but also in solution, as shown with two observed ${ }^{31} \mathrm{P}\left\{{ }^{1} \mathrm{H}\right\}$ NMR singlets at $\delta 18.47$ and $27.77 \mathrm{in}$

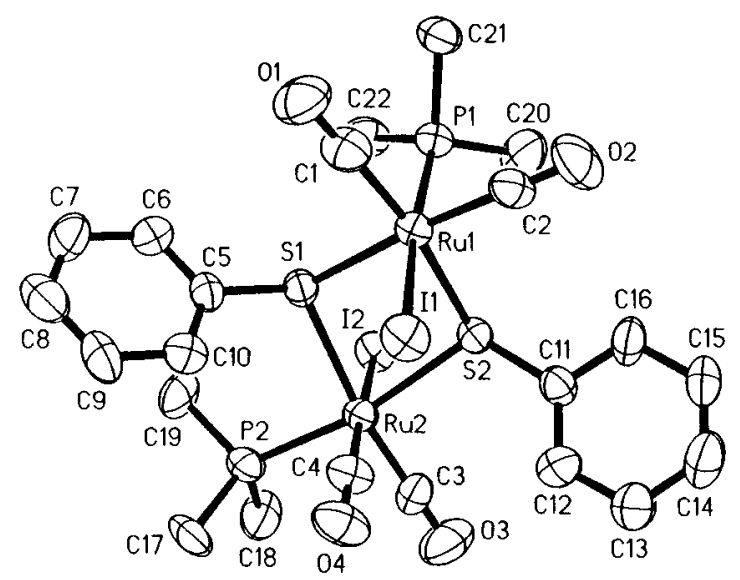

Figure 5. ORTEP plot of $\left[\mathrm{Ru}_{2}(\mathrm{CO})_{4}(u-\mathrm{SPh})_{2} \mathrm{I}_{2}\left(\mathrm{PMe}_{3}\right)_{2}\right](\mathbf{1 0})$. Selected bond distances $(\AA)$ : $R u(1)-I(1)=2.799(1)$, Ru$(1)-\mathrm{S}(1)=2.466(1), \mathrm{Ru}(1)-\mathrm{S}(2)=2.458(1), \mathrm{Ru}(1)-\mathrm{P}(1)=$ 2.337(1), Ru(1)-C(1) = 1.886(6), Ru(1)-C(2) = 1.869(6), $\mathrm{Ru}(2)-\mathrm{S}(1)=2.471(1), \mathrm{Ru}(2)-\mathrm{S}(2)=2.463(1), \mathrm{Ru}(2)-\mathrm{P}(2)$ $=2.360(1), R u(2)-C(3)=1.865(9), R u(2)-C(4)=1.859(7)$. Selected bond angles (deg): I(1)-Ru(1)-P(1) = 171.6(1), $\mathrm{I}(2)-\mathrm{Ru}(2)-\mathrm{C}(4)=172.7(2), \mathrm{Ru}(1)-\mathrm{S}(1)-\mathrm{Ru}(2)=98.6(1)$, $\mathrm{Ru}(1)-\mathrm{S}(2)-\mathrm{Ru}(2)=99.1(1), \mathrm{P}(1)-\mathrm{Ru}(1)-\mathrm{S}(1)=91.5(1)$, $\mathrm{P}(1)-\mathrm{Ru}(1)-\mathrm{S}(2)=90.2(1), \mathrm{P}(2)-\mathrm{Ru}(2)-\mathrm{S}(1)=94.5(1)$, $\mathrm{P}(2)-\mathrm{Ru}(2)-\mathrm{S}(2)=168.3(1)$.

\section{Scheme 3}
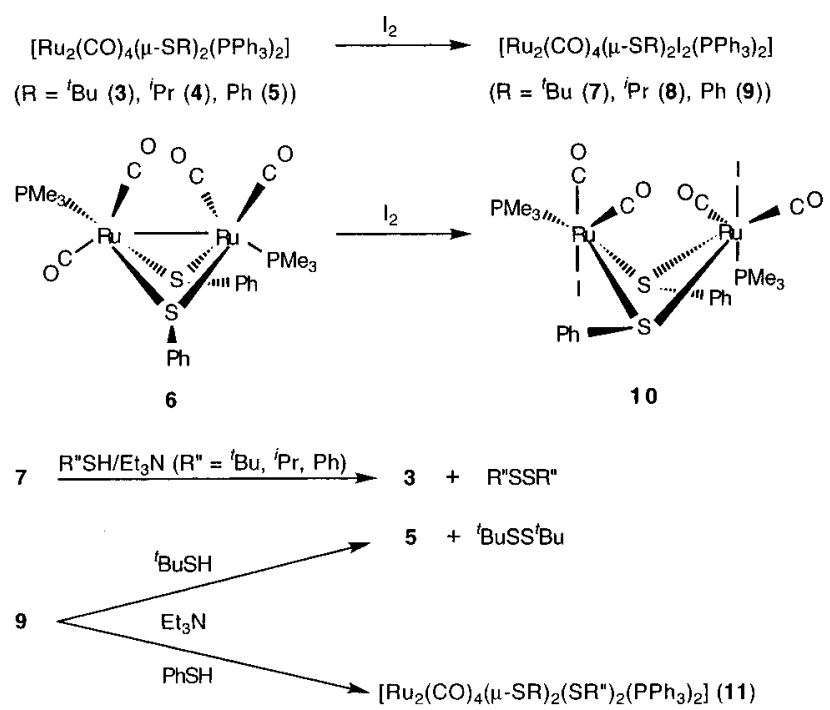

acetone- $d_{6}$ at 300 and $220 \mathrm{~K}$. The long distance with $\mathrm{d}(\mathrm{Ru} \cdots \mathrm{Ru})=3.743 \AA$ between two $\mathrm{Ru}$ atoms indicates no Ru-Ru single bond in $\mathbf{1 0}$ and probably also in 7-9. The two iodides are trans to each other, reflecting a trans addition of di iodine to $\mathbf{6}$ and probably also to 3-5. If the resulting structure 7-9 has two trans iodide atoms with each at one Ru atom, the observed ${ }^{31} \mathrm{P}\left\{{ }^{1} \mathrm{H}\right\}$ NMR singlet at $\delta 44.58$ for 7, 46.65 for $\mathbf{8}$, and 50.97 for 9 in acetone- $d_{6}$ at 300 and $220 \mathrm{~K}$ may suggest a very facile process, probably involving conversion of two terminal into bridging iodides concomitantly with opening two bridging into terminal thiolato groups while not permitting rotation of two carbonyls and one phosphine attached to each $\mathrm{Ru}$ atom along a pseudo- $\mathrm{C}_{3}$ axis (Scheme 4), resulting in two equivalent and inequivalent phosphine atoms for 7-9 and $\mathbf{1 0}$, respectively, at the NMR time scale. The evidence of the three-band $v_{\text {co }}$ pattern and the one ${ }^{31} \mathrm{P}\left\{{ }^{1} \mathrm{H}\right\}$ NMR singlet described for 
Scheme 4

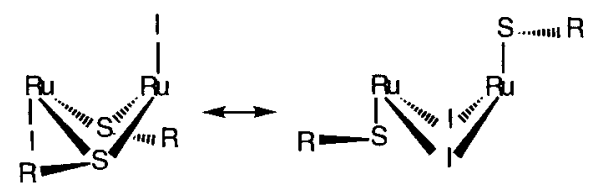

7-9 and that of one ${ }^{1} \mathrm{H}$ NMR singlet at $\delta 0.76$ for the two tBu groups in 7, one doublet at 0.95 and one heptet at 2.99 for two ${ }^{i} P r$ groups in 8, and one ${ }^{31} \mathrm{C}$ NMR doublet at 192.5 with J P,C $=11.0 \mathrm{~Hz}^{13}$ for the four carbonyl groups in 9 suggest that 7-9 should adopt probably the syn form rather than the anti form.

The reported reaction chemistry of $\left[\mathrm{Fe}_{2}(\mathrm{CO})_{4}(\mu-\mathrm{SR})_{2^{-}}\right.$ $\left.\left(\mathrm{PPh}_{3}\right)_{2}\right](\mathrm{R}=\mathrm{Et}, \mathrm{Ph})$ with $\mathrm{X}_{2}(\mathrm{X}=\mathrm{Cl}, \mathrm{Br}, \mathrm{I})$ is quite rich, producing either $\left[\mathrm{Fe}_{2}(\mathrm{CO})_{4}(u-\mathrm{SR})_{2}\left(\mathrm{PPh}_{3}\right)_{2} \mathrm{l}\right] \mathrm{l}_{3}$ or $\left[\mathrm{Fe}_{3}(\mathrm{CO})_{4}(u-\mathrm{SR})_{2}\left(\mathrm{PPh}_{3}\right)_{2} \mathrm{X}_{4}\right] \mathrm{X}$ with noFe-Femetal bond. ${ }^{4 b}$ However, decarbonylation occurs quite readily for iodination of 7-9, resulting in intractable products, apparently without carbonyl groups attached to the metal center on the basis of the IR spectra obtained. Reactions of 7-9 with nucleophiles such as thiolate anions were then carried out. The reaction results between complexes 7 and 9 and the anions via R"SH/Et ${ }_{3} N\left(R^{\prime \prime}=t_{-}\right.$ $\mathrm{Bu},{ }^{i} \mathrm{Pr}, \mathrm{Ph}$ ) appear somewhat unexpectedly to have two reaction pathways. Among the five reactions studied, only the one between 9 and $\mathrm{PhS}^{-}$gave the expected substitution product, $\left[\mathrm{Ru}_{2}(\mathrm{CO})_{4}(\mu-\mathrm{SPh})_{2}(\mathrm{SPh})_{2}\left(\mathrm{PPh}_{3}\right)_{2}\right]$ (11). Other reactions regenerated reductively $\mathbf{3}$ and $\mathbf{5}$ with the apparently external R"S anions being oxidized into R"SSR" in high yield (Scheme 3). Disulfide formation was previously reported for the internally ligated thi olato groups of $\left[\mathrm{Fe}_{2}(\mathrm{CO})_{6}(u-\mathrm{SR})_{2}\right]$ but under oxidation conditions with $\mathrm{O}_{2}$, ${ }^{4 j}$ different from our examples. Under similar reaction conditions with excess $\mathrm{R}^{\prime \prime} \mathrm{SH} /$ $\mathrm{Et}_{3} \mathrm{~N}$ relative to the complex (e.g., $1 \mathrm{~mL}$ of $\mathrm{R}^{\prime \prime} \mathrm{SH}, 2 \mathrm{~mL}$ of $\mathrm{Et}_{3} \mathrm{~N}$, and ca. $0.30 \mathrm{mmol}$ of the complex), we found that the time required for a complete redox reaction is ca. $2 \mathrm{~h}$, much longer than that of $0.5 \mathrm{~h}$ needed for a complete substitution reaction. Further, on the basis of the IR spectra measured sequentially for the longer redox reaction, no intermediate compounds were observed with typical IR spectra recorded in an overlaid mode for the reaction between 7 and $\mathrm{PhSH} / \mathrm{Et}_{3} \mathrm{~N}$ in $\mathrm{CH}_{2-}$ $\mathrm{Cl}_{2}$. These facts led us to propose a mechanism favoring substitution (routea) rather than a redox process (route b) for the first step of the reaction. ${ }^{15}$ It probably involves the replacement of one iodide at the less sterically congested site (cf. Scheme 5) with one thiolate anion to form the intermediate $\left[\mathrm{Ru}_{2}(\mathrm{CO})_{4}(u-\mathrm{SR})_{2}\left(\mathrm{SR}^{\prime \prime}\right) \mid\left(\mathrm{PR}_{3}{ }_{3}\right)_{2}\right]$. $\mathrm{F}$ or $\mathrm{PhS}^{-}$, the steric hindrance of this intermediate with $R=R^{\prime}=R^{\prime \prime}=P h$ is not large, enabling a further replacement reaction (route c) to give $\mathbf{1 1}$. However, when the hindrance of the intermediate with one of the other combinations of $R, R^{\prime}$, and $R^{\prime \prime}$ (i.e., $R=R^{\prime}=P h$, $\mathrm{R}^{\prime \prime}=\mathrm{t}^{\mathrm{Bu}}$; or $\mathrm{R}=\mathrm{Ph}, \mathrm{R}^{\prime}={ }^{\mathrm{t}} \mathrm{Bu}, \mathrm{R}^{\prime \prime}=\mathrm{Ph}$, $\mathrm{Pr}$, or ${ }^{\mathrm{t}} \mathrm{Bu}$ ) is too high to allow a second replacement reaction, a redox reaction (routed) occurs with regeneration of complexes $\mathbf{3}$ and $\mathbf{5}$ and R"SSR".

Like 9, 11 displays one ${ }^{31} \mathrm{P}\left\{{ }^{1} \mathrm{H}\right\}$ NMR singlet for the two $\mathrm{PPh}_{3}$ ligands in this compound dissolved in $\mathrm{CH}_{2-}$

(13) The coupling constant is compatible with the reported values. ${ }^{14}$

(14) Gill, D. F.; Mann, B. E.; Shaw, B. L. J . Chem. Soc., Dalton Trans. 1973, 311.

(15) Astruc, D., Ed. Electron Transfer and Radical Processes in Transition-Metal Chemistry; VCH: New York, 1995.

\section{Scheme 5}
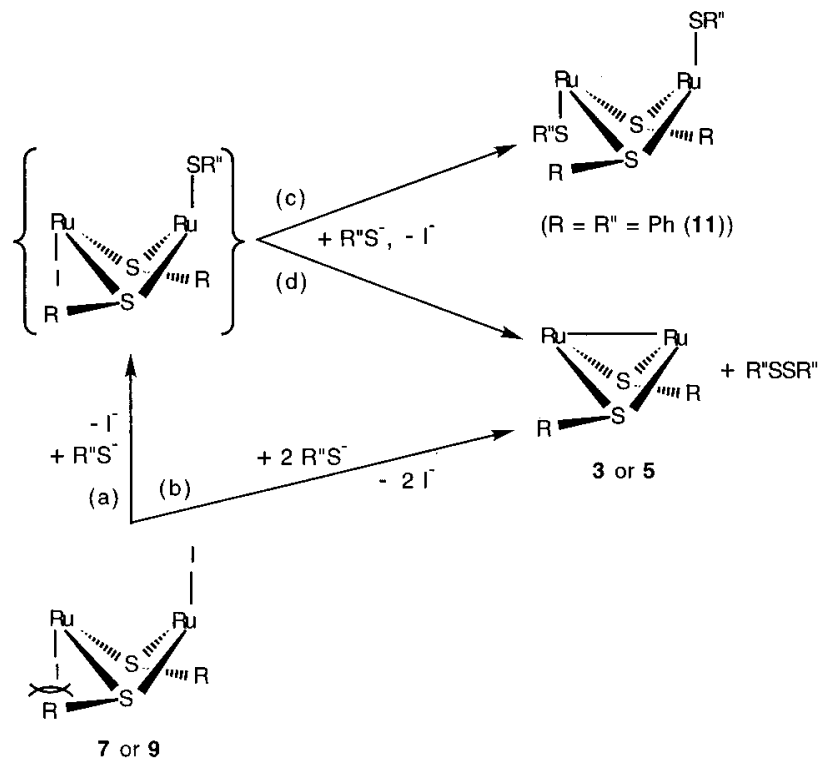

$\mathrm{Cl}_{2}$ at 300 or $220 \mathrm{~K}$, suggesting a similar facile process involving conversion of two terminal into bridging thiolato groups concomitantly with opening two bridging into terminal thiolato groups (cf. Scheme 4) to make two phosphine atoms equivalent at the NMR time scale. However, the two structures are different, with a syn form for $\mathbf{9}$ and an anti form for 11, based on the two observed ${ }^{13} \mathrm{C}$ NMR doublets at $\delta 197.2(\mathrm{~J}=8.3 \mathrm{~Hz})^{13}$ and $197.5(\mathrm{~J}=9.7 \mathrm{~Hz})^{13}$ for the four carbonyls in this compound dissolved in $\mathrm{CDCl}_{3}$. Apparently, it is rather difficult to predict the structure preference for any diruthenium compounds with two thiol ato bridges prior to examining the characterization data. The two thiolato bridges in a dinuclear system may adopt a syn form like $\mathbf{3}$ and $\mathbf{7 - 1 0}$ or an anti form like $\mathbf{6}$ and $\mathbf{1 1}$, or they may involve an equilibrium mixture of syn and anti forms with the syn form favored for $\mathbf{4}$ and the anti form for $\mathbf{5}$ in solution. This nonspecific feature is probably more associated with steric effects than electronic effects, and the one or two forms adopted may represent nonbonded interactions of low, if not the least, repulsiveness.

\section{Conclusion}

Our investigation into diruthenium carbonyl phosphine complexes containing two $\mu$-thiolato linkages resulted in the facile synthesis of 3-6 from $\mathbf{1}$ and $\mathbf{2}$ in satisfactory yield (Scheme 1). Electrophilic additions of 3-6 with $\mathrm{I}_{2}$ produce di iodide adducts 7-10. Further reactions of $\mathbf{7}$ and $\mathbf{9}$ with thiolate anions give either expected nucleophilic substitution products such as $\mathbf{1 1}$ or unexpected reductive-dehalogenation to regenerate complexes $\mathbf{3}$ and 5, respectively, with the apparently external anions being oxidized into alkyl or aryl disulfides (Scheme 3). Various spectral data and five X-ray crystal structures (Figures 1-5) help to establish that the two thi olato bridges in 3-11 may adopt a syn form or an anti form, or they may involve an equilibrium mixture of both forms with one form or the other favored in solution. An unprecedented structure type with the two thiolato bridges located cis to one of two phosphine ligands in the compounds but trans to the other was 
observed in 6 (Figure 4 ) and retained in the iodination product $\mathbf{1 0}$ (Figure 5), in spite of the anti geometry in $\mathbf{6}$ being changed into syn in $\mathbf{1 0}$.

Acknowledgment. Financial support for this work by the National Science Council of Republic of China (Contract NSC87-2113-M006-007) and skillful assistance by Ms. Fang-Chung Chou are gratefully acknowledged.
Supporting Information Available: Tables of nonhydrogen atomic coordinates and equivalent isotropic displacement coefficients, complete bond lengths and angles, anisotropic displacement coefficients, and hydrogen coordinates for $\mathbf{3}, \mathbf{4 A}, \mathbf{5 B}, \mathbf{6}$, and $\mathbf{1 0}$ (30 pages). Ordering information is given on any current masthead page.

OM9710024 